\title{
Impact of Curcuma longa extract on the expression level of brain transporters in in vivo model
}

\author{
MARTA BUKOWSKA ${ }^{1}$, ANNA BOGACZ ${ }^{1,2^{*}}$, MARLENA WOLEK ${ }^{3}$, PRZEMYSŁAW Ł. \\ MIKOŁAJCZAK ${ }^{2,4}$, PIOTR OLBROMSKI ${ }^{5}$, ADAM KAMIŃSKI ${ }^{6}$, BOGUSŁAW CZERNY ${ }^{3,7}$
}

\author{
${ }^{1}$ Department of Histocompatibility with Laboratory of Genetic Diagnostics \\ Regional Blood Center \\ Marcelińska 44 \\ 60-354 Poznań, Poland \\ ${ }^{2}$ Department of Pharmacology and Phytochemistry \\ Institute of Natural Fibres and Medicinal Plants \\ Kolejowa 2 \\ 62-064 Plewiska, Poland \\ ${ }^{3}$ Department of Stem Cells and Regenerative Medicine \\ Institute of Natural Fibres and Medicinal Plants \\ Kolejowa 2 \\ 62-064 Plewiska, Poland \\ ${ }^{4}$ Department of Pharmacology \\ Poznan University of Medical Sciences \\ Rokietnicka 5a \\ 60-806 Poznań, Poland \\ ${ }^{5}$ Clinic of Operational Gynecology \\ Poznan University of Medical Sciences \\ Polna 33 \\ 60-535 Poznań, Poland \\ ${ }^{6}$ Clinic of Pediatric Orthopedics \\ Pomeranian Medical University \\ Unii Lubelskiej 1 \\ 62-600 Szczecin, Poland \\ ${ }^{7}$ Department of Pharmacology and Pharmacoeconomics \\ Pomeranian Medical University \\ Żołnierska 48 \\ 71-230 Szczecin, Poland
}

*corresponding author: aniabogacz23@o2.pl 


\section{Summary}

Introduction: Blood brain barrier and multidrug resistance phenomenon are subjects of many investigations. Mainly, because of their functions in protecting the central nervous system (CNS) by blocking the delivery of toxic substances to the brain. This special function has some disadvantages, like drug delivery to the brain in neurodegenerative diseases.

Objective: The aim of this study was to examine how natural and synthetic substances affect the expression levels of genes (Mdr1a, Mdr1b, Mrp1, Mrp2, Oatp1a4, Oatp1a5 and Oatp1c1) that encode transporters in the blood-brain barrier.

Methods: cDNA was synthesized from total RNA isolated from rat hippocampus. The expression level of genes was determined using real-time PCR (RT-PCR) method.

Results: Our findings showed that verapamil, as a synthetic substance, caused the greatest reduction of mRNA level of genes studied. The standardized extract of Curcuma longa reduced the expression level for Mrp1 and Mrp2, whereas the increase of mRNA level was observed for Mdr1b, Oatp1a5 and Oatp1c1.

Conclusions: These results suggests that herbal extracts may play an important role in overcoming the blood brain barrier during pharmacotherapy.

Key words: blood brain barrier, Curcuma longa, expression level, natural and synthetic substances

Słowa kluczowe: bariera krew-mózg, Curcuma longa, poziom ekspresji, substancje naturalne

i syntetyczne

\section{INTRODUCTION}

Brain diseases might take various forms, such as infections, trauma, stroke, seizures and tumors. Brain inflammations can be caused by viral and bacterial infections [1]. Epilepsy is a brain disease categorized as a seizure condition, which is characterized by abnormal brain's electrical activity [2]. Trauma is a brain injury that may cause a temporary or a permanent disturbance in brain function [3]. Tumors are abnormal cells that form tissues and grow inside the brain. Whether malignant or benign, tumors are very dangerous due to the pressure they exert on an unchanged brain tissue [4]. Finally, stroke is a brain disease connected with the condition of blood vessels. During stroke, blood and oxygen flow are suddenly disturbed. Areas of brain that weren't properly supplied by blood and oxygen might be permanently damaged. Body parts which are controlled by those damaged brain areas might no longer work correctly - or even at all [5].

Even though the forms of brain diseases differ in etiology or symptoms, most of them lead to cell death [6-8]. Due to the great importance of brain, such as neural signaling within central nervous system (CNS), it requires microenvironment that would be highly controlled. There are three forms of protection between blood and CNS: the blood barrier (BBB), the blood-CSF barrier and also the arachnoid barrier [9]. The blood barrier is a selective membrane. BBB creates a chemical and physical barrier between blood and brain parenchyma. As to specialized cells and transporters that the $\mathrm{BBB}$ has, it can regulate the environment of CNS [6]. Thanks to those regulations, CNS may function properly [10]. The homeostasis of CNS might be changed by morphological or functional disturbances that can result in brain damage $[11,12]$. As the BBB's main role is to stop the transports of majority of substances to the brain, tightness of this barrier is variable. The $\mathrm{BBB}$ contains numerous transporters and channels that allow to be transported nutrients needed to proper functioning of the brain [6]. The BBB also contains ion transporters and channels that appear to be promising as a therapeutic path to be challenged.

There are many studies that explore usefulness of transporters as a drug deliver paths in brain diseases [11]. Transporters expressed by CNS may be divided into two types: efflux and nutrient. Firstly, the efflux transporters (e.g. MDR1, BCRP, MRPs) use the ATP's hydrolysis to transport their substrates up the concentration gradient. While the nutrient transporters simplify the transport of the specific substances down the concentration gradient (e.g. slc2a1, slc16a1, slc7a1, slc7a). Despite the 
fact that those transporters mainly provide substances to the brain, some of them are removing waste and toxic products from the brain. In brain diseases, all of the protective functions of the $\mathrm{BBB}$ are weaken $[10,13,14]$.

For many years, neurological diseases were only diagnosed and the treatment was almost impossible. Nowadays, the neurology itself blossoms and it gives a hope to find new drugs and ways to deliver them [8, 15]. It is believed that new drug programmes should consider the special features of the BBB [10]. Unfortunately, most of clinical substances commonly used in brain diseases provide narrow improvement and are accompanied by side effects. Despite all difficulties, treatment of CNS diseases still remains a major goal for scientists [16]. New studies show how interesting is the effect of the substances of herbal origin on the expression of transporter gene conditioning, the blood brain barrier and phenomenon of multidrug resistance (MDR). Most of studies focus on P-gp expression and the impact of synthetic and herbal substances. Verapamil is one of the synthetic substances taken into consideration as a P-gp inhibitor. Unfortunately, due to cardiac toxicity, verapamil cannot be allowed in practice. However, the inhibitors of herbal origin seem to have less side effects on normal tissues than synthetic substances. Furthermore, some flavonoid compounds, such as kaempferol, daidzein, quercetin, genistein, may decrease the P-gp pump's activity. The mechanism is based on the reduction of the resistance of selected cell lines to vinblastin. Curcumin (from rhizomes of Curcuma longa L., Zingiberaceae), for example, is believed to decrease the P-gp's activity $[17,18]$.

Consequently, the main aim of this paper was to analyze the effects of synthetic and herbal substances, which might be important in blocking the function of transporters in blood-brain barrier.

\section{EXPERIMENTAL}

\section{Study design}

Due to its inhibitory properties, in this experiment verapamil was used as a standard chemical compound. Some of synthetic (verapamil and piracetam) and herbal substances (quercetin, codeine, cyclosporine A) were purchased from SigmaAldrich Chemical Company (USA), while phenobarbital was obtained from PGF Cefarm-Poznan Sp. Z. o. o. (Poland). The rhizomes of Curcuma longa L., Zingiberaceae, were used as a standardized extracts and were purchased from Finzelberg $\mathrm{GmbH} \& \mathrm{Co}$. KG (Germany). High performance liquid chromatography (HPLC), as a procedure included in "European Pharmacopoeia", was used to determine the content of active compounds of selected dry alcoholic extracts (86\% curcuminoids, $7 \%$ essential oils). Designed experiment was performed on male Wistar rats (300-450 g). All tests were performed in accordance with Polish governmental regulations and in agreement with Local Ethics Committee on the Use of Laboratory Animals in Poznań, Poland (No. 16/2010). The experiment was performed at the Department of Pharmacology, Poznan University of Medical Sciences. The rats were kept in plastic cages, climate-controlled room with a $12 \mathrm{~h}$ light/dark cycles and full access to rat chow and tap water. Animals were divided into 8 groups from I to VIII, with 8 rats per group. For 21 days, all rats were receiving herbal extracts, synthetic and natural substances were suspended in water. Further details of the treatment are described in table 1. Group VIII was used as a control group. Rats included in group VIII were fed only standard diet. Wistar rats were decapitated two hours after the last administration

Table 1

List of individual groups of rats depending on the substances used

\begin{tabular}{lll}
\hline Group & Substance & Dose \\
\hline I & Verapamil & $20 \mathrm{mg} \mathrm{kg}^{-1}$ per day, $p . o$. \\
\hline II & Cyclosporine A & $5 \mathrm{mg} \mathrm{kg}^{-1}$ per day, $p . o$. \\
\hline III & Piracetam & $200 \mathrm{mg} \mathrm{kg}^{-1}$ per day, $p . o$. \\
\hline IV & Phenobarbital & $80 \mathrm{mg} \mathrm{kg}^{-1}$ per day, $p . o$. \\
\hline V & Quercetin & $500 \mathrm{mg} \mathrm{kg}^{-1}$ per day, $p . o$. \\
\hline VI & Codeine & $20 \mathrm{mg} \mathrm{kg}^{-1}$ per day, $p . o$. \\
\hline VII & Rhizome extract of Curcuma longa & $500 \mathrm{mg} \mathrm{kg}^{-1}$ per day, $p . o$. \\
\hline VIII & None & Control \\
\hline
\end{tabular}


of particular substance. The obtained hippocampus were frozen in liquid nitrogen and preserved at $-80^{\circ} \mathrm{C}$.

\section{Expression analysis}

In this paper, we studied the effect of seven substances that may play a role in blocking functions of MDR1 (multidrug resistance protein 1), MRP1 (multidrug resistance-associated protein 1), MRP2 (multidrug resistance-associated protein 2), OATP1A4, OATP1A5 and OATP1C1 (SLC transporters). In the experiment, the total cellular RNA was isolated from rat hippocampus. The RNA was isolated using TriPure Isolation Reagent from Roche (Germany). BioPhotometer by Eppendorf (USA) was used to determinate the RNA's concentration and purity. According to manufacturer's protocol, complementary DNA was synthesized from $1 \mu \mathrm{g}$ of RNA in volume of $20 \mu \mathrm{l}$. To obtain cDNA we used Transcriptor cDNA First Strand Synthesis Kit and oligo(dT) 18 primer by Roche. According to manufacturer's protocol, LightCycler 480 instrument (Roche) and a LightCycler ${ }^{\circ} 480$ SYBR Green I Master (Roche) were used to carry out the real-time PCR (RT-PCR). We confirmed the amplicon size and specificity of the reaction by agarose electrophoresis and melting curve analysis. For normalization, as a housekeeping gene (endogenous internal standard), we used GAPDH cDNA. The primers and the conditions of reactions were used according to
Mrozikiewicz et al. [17]. The LightCycler 480 Basic Software by Roche was used to evaluate data (Roche Applied Science, Berlin, Germany).

\section{Statistical analysis}

To express the mRNA content of the examined genes, mean \pm SEM was used. Obtained data were analysed using the SPSS 17.0 for Windows software. We compared the mean values to means of the oneway ANOVA test. As the statistically significant value, we considered the value of $p<0.05$

\section{RESULTS}

The aim of this paper was to analyse the influence of natural, synthetic substances and also herbal extracts on the level of gene expression that code transporters involved in $\mathrm{BDB}$ and MDR (multidrug resistance).

The study's results showed that administration of codeine and also piracetam caused a decrease of the expression in all investigated genes encoding transporters aside from Oatp1c1. Administration of cyclosporin A significantly decreased the mRNA level of Mdr1a, Mrp1 and Mrp2. We also noticed reduction of expression level for Oatpla4, but it was not a significant decrease. Verapamil as an inhibitor of xenobiotic transporters decreased the expression level in all examined genes. In contrary to verapamil, administration of phenobarbital

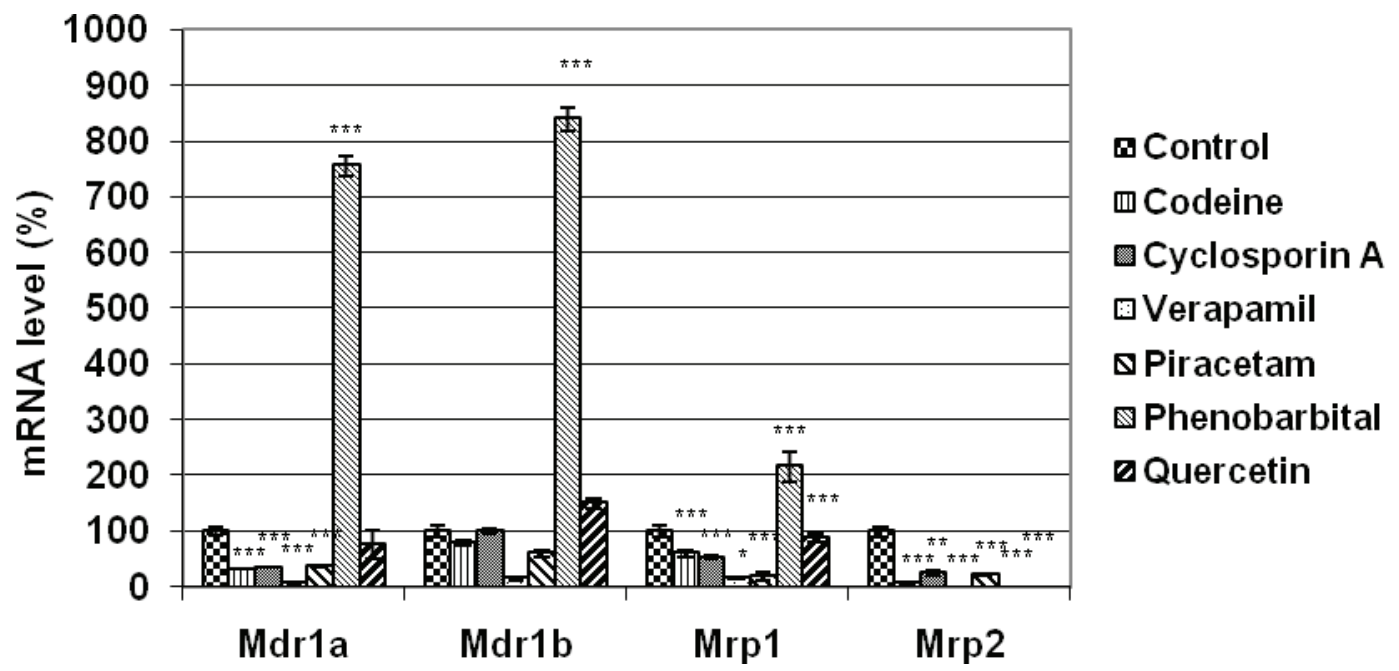

Figure 1

The expression levels of genes encoding transmembrane transporters (Mdr1a, Mdr1b, Mrp1, Mrp2) in the rat's hippocampus after 21 days of administration of synthetic and natural substances. Control groups are defined as $100 \%$. Data are presented as mean \pm SEM. Asterisks indicate significance (one-way ANOVA test): ${ }^{\star} p<0.05,{ }^{* *} p<0.001,{ }^{* *} p<0.0001$. 


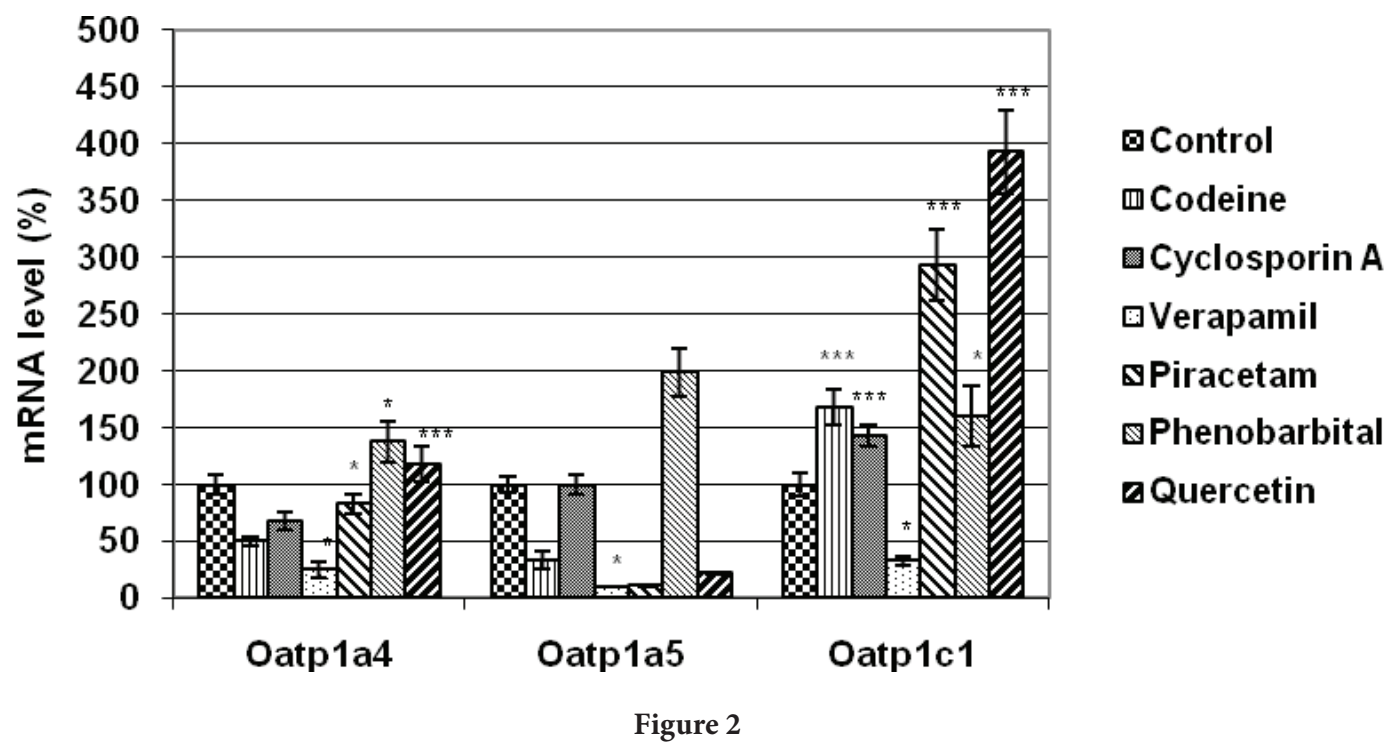

The expression levels of genes encoding transmembrane transporters (Oatp1a4, Oatp1a5, Oatp1c1) in the rat's hippocampus after 21 days of administration of synthetic and natural substances. Control groups are defined as $100 \%$. Data are presented as mean \pm SEM. Asterisks indicate significance (one-way ANOVA test): ${ }^{\star} p<0.05,{ }^{* *} p<0.001,{ }^{* * *} p<0.0001$.

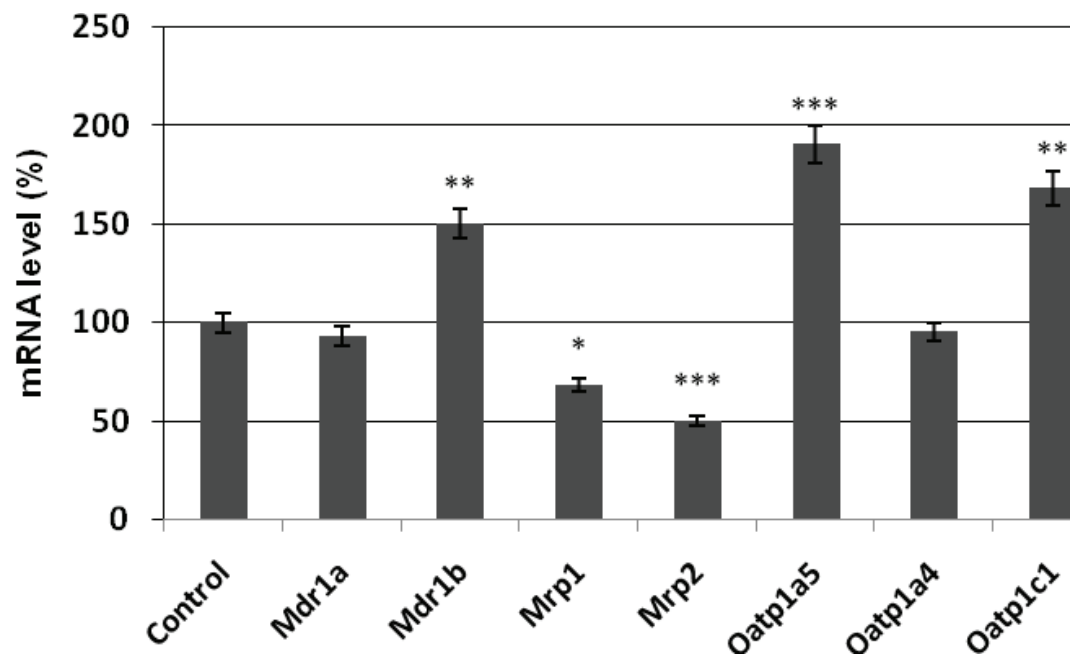

Figure 3

The expression levels of genes encoding transmembrane transporters (Mdr1a, Mdr1b, Mrp1, Mrp2, Oatp1a4, Oatp1a5, Oatp1c1) in the rat's hippocampus after 21 days of administration of Curcuma longa extract. Control groups are defined as $100 \%$. Data are presented as mean \pm SEM. Asterisks indicate significance (one-way ANOVA test): ${ }^{*} p<0.05,{ }^{* *} p<0.001,{ }^{* * *} p<0.0001$

resulted in an increased expression level of all investigated genes except for Mrp2. Quercetin had an inductive effect on the expression level for Mdrlb, Oatpla4 and Oatplc1 but also decreased the mRNA level for Mrp2 (fig. 1, 2).

Interesting results were obtained in the herbal extract. The study showed that $C$. longa increased the expression level for Mdr1b (50\%), Oatp1a5 (90\%) and Oatp1c1 (68\%). However, the administration of $C$. longa extract caused a decrease of the mRNA level for Mrp2 (50\%) and Mrp1 (32\%) (fig. 3).

\section{DISCUSSION}

Due to specific properties of blood-brain barrier, drug delivery in brain diseases often fails. Drug molecules fail to cross BBB due to their poor penetration or/and to the efflux mechanism of the brain. Nowadays, distribution of drugs to CNS across the $\mathrm{BBB}$ is one of the most challenging problems in pharmacy [19]. More and more scientists are focusing on finding perfect ways to inhibit active transporters that are involved in functioning of the $\mathrm{BBB}$ and MDR phenomenon [17]. 
Some of the researchers worked on Panax ginseng, Hypericum perforatum or Ginkgo biloba to show changes in mRNA level. Zhang et al. showed that administration of $P$. ginseng caused induction of P-gp expression in intestinal and brain endothelium [20]. Moreover, it also increased the level of Mrp2 mRNA in rat primary hepatocytes [21]. According to Mrozikiewicz et al., $P$. ginseng extract may be useful in overcoming blood brain barrier and multidrug resistance phenomenon. $H$. perforatum seems to increase the expression level Mdr1a, Mdrlb in brain, liver, kidney, heart and also increases the expression level of Mrp2 in rat livers and kidneys [17, 22]. Furthermore, studies conducted on G. biloba showed that its extract can increase the expression level for Mdr1, Oatp1a4, Oatpla5. What is interesting, the G. biloba extract decreased the mRNA level for Mrp2, which may be helpful in CNS diseases with treatment based on substrates for Mrp2 [16, 17, 23].

In this study, the influence that $C$. longa may have on the mRNA levels for particular transporters was analysed. The results suggests that using $C$. longa extract in the treatment of brain diseases would be effective in case of drugs which are substrates for Mrp2 and Mrp1. Yuan et al. studied mechanisms underlying subarachnoid hemorrhage $(\mathrm{SAH})$ and examined how treatment based on C. longa extract may ameliorates $\mathrm{SAH}$-induced brain oedema and BBB permeability changes. According to Yuan et al., curcumin may inhibit matrix metallopeptidase- 9 expression. Decreased expression of metallopeptidase- 9 reduces brain oedema and attenuates post-SAH BBB disruption in mice [24].

Wang et al. examined if $C$. longa extract has any effect on the disruption of BBB which may be induced by brain ischaemia. Oxygen glucose deprivation(OGD)-induced disruption of paracellular permeability was weaken by $C$. longa extract. The extract also increased the expression of heme oxygenase-1 (HO-1) protein in rat brain microvascular endothelial cells (RBMECs). According to this paper, C. longa extract protects RBMECs against OGD-induced dysfunction of BBB thanks to the HO-1 pathway. It may mean that, under ischaemic conditions, the herbal extract may be capable of improving the barrier function of $\mathrm{BBB}$. What is also interesting, that beneficial effect can be reversed by a HO-1 inhibitor [25].

Klinger and Mittal have a different opinion about C. longa. They wondered if C. longa can have any therapeutic potential during brain tumours treatment. Due to its antioxidant, anti-inflammatory, and antiproliferative properties, Curcuma longa is a promise in pharmacy. They focused on therapeutic features of Curcuma longa, maintaining that distribution of the extract to the brain might be hindered by limited BBB permeability. They showed that only adding particular nanoparticle formulations, e.g. poly(lacticco-glycolic acid), may increase its distribution to the brain tissue [26].

\section{CONCLUSION}

Overcoming blood brain barrier to deliver drugs is still a challenge for modern scientists. As the studies show, herbal origin and synthetic drugs can modulate expression of transporters in $\mathrm{BBB}$. The synthetic substance that decreased the mRNA level most was verapamil. The herbal extract we examined was obtained from C. longa and also reduced the mRNA level but only for Mrp2 (50\%) and Mrp1 (32\%). Those results might suggest that verapamil is a better inhibitor and should be used in overcoming BBB. But we should take into account that using herbal extract does not come with side effects as it comes with verapamil. In conclusion, using herbal extracts may have an interesting potential in pharmacotherapy of brain diseases.

\section{ACKNOWLEDGEMENTS}

This research was supported by the Polish Ministry of Science and Higher Education, Grant No. N405 677140.

Conflict of interest: Authors declare no conflict of interest.

\section{REFERENCES}

1. Sarrazin JL, Bonneville F, Martin-Blondel G. Brain infections. Diagn Interv Imaging 2012; 93(6):473-90. doi: http://dx.doi.org/10.1016/j. diii.2012.04.020

2. Fisher RS, Acevedo C, Arzimanoglou A, Bogacz A, Cross JH, Elger CE, et al. ILAE official report: a practical clinical definition of epilepsy. Epilepsia 2014; 55(4):475-82. doi: http:// dx.doi.org/ 10.1111/epi.12550

3. Saatman KE, Duhaime AC, Bullock R, Maas 
AIR, Valadka A, Manley GT, et al. Classification of traumatic brain injury for targeted therapies. J Neurotrauma 2008; 25(7):719-38. doi: http:// dx.doi.org/10.1089/neu.2008.0586

4. McFaline-Figueroa JR, Lee EQ. Brain Tumors. Am J Med 2018; 131(8):874-882. doi: http:// dx.doi.org/10.1016/j.amjmed.2017.12.039

5. Seifert HA, Offner $\mathrm{H}$. The splenic response to stroke: from rodents to stroke subjects. J Neuroinflammation 2018; 5:195. doi: http://dx.doi. org/10.1186/s12974-018-1239-9

6. Florendo M, Figacz A, Srinageshwar B, Sharma A, Swanson D, Dunbar GL, et al. Use of polyamidoamine dendrimers in brain diseases. Molecules 2018; 23(9):2238. doi: http://dx.doi.org/10.3390/ molecules 23092238

7. Chen M, Du ZY, Zheng X, Li DL, Zhou RP, Zhang $\mathrm{K}$. Use of curcumin in diagnosis, prevention, and treatment of Alzheimer's disease. Neural Regen Res 2018; 13(4):742-752. doi: http://dx.doi. org/10.4103/1673-5374.230303

8. Mishra S, Palanivelu K. The effect of curcumin (turmeric) on Alzheimer's disease: An overview. Ann Indian Acad Neurol 2008; 11(1):13-19. doi: http://dx.doi.org/10.4103/0972-2327

9. Abbott NJ, Patabendige AA, Dolman DE, Yusof SR, Begley DJ. Structure and function of the blood-brain barrier. Neurobiol Dis 2010; 37(1):13-25. doi: http://dx.doi.org/10.1016/j. nbd.2009.07.030

10. Daneman R, Prat A. The blood-brain barrier. Cold Spring Harb Perspect Biol 2015; 7(1):a020412. doi: http://dx.doi.org/10.1101/cshperspect.a020412

11. Moretti R, Chhor V, Titomanlio L, Fleiss B, Gressens P. Brain edema in developing brain diseases. In: Brain edema. From molecular mechanisms to clinical practice. London 2017:393-429.

12. Tajes $M$, Ramos-Fernández E, Weng-Jiang $X$, Bosch-Morató M, Guivernau B, Eraso-Pichot A, et al. The blood-brain barrier: structure, function and therapeutic approaches to cross it. Mol Membr Biol 2014; 31(5):152-67. doi: http://dx.doi.org/ 10.3109/09687688.2014.937468
13. Schenk GJ, Kooij G, Reijerkerk A, de Vries H. Disease influence on BBB transport in inflammatory disorders. In: Hammarlund-Udenaes $\mathrm{M}$, de Lange E, Thorne R. (eds.). Drug delivery to the brain. AAPS advances in the pharmaceutical sciences. New York 2014, 10:573-589.

14. Bogacz A, Deka-Pawlik D, Bartkowiak-Wieczorek J, Karasiewicz M, Kujawski R, Kowalska A, et al. The effect of herbal materials on the P-glycoprotein activity and function. Herba Pol 2013; 59(4):129-141. doi: http://dx.doi.org/10.2478/ hepo-2013-0029

15. Young AB. Four decades of neurodegenerative disease research: How far we have come! J Neurosci 2009; 29(41):12722-12728. doi: http://dx.doi. org/10.1523/JNEUROSCI.3767-09.2009

16. Zhang F, Lin Y, Kannan S, Kanan RM. Targeting specific cells in the brain with nanomedicines for CNS therapies. J Control Release 2016; 240:212-226. doi: http://dx.doi.org/10.1016/j. jconrel.2015.12.013

17. Mrozikiewicz PM, Bogacz A, Bartkowiak-Wieczorek J, Kujawski R, Mikołajczak PL, et al. Screening for impact of popular herbs improving mental abilities on the transcriptional level of brain transporters. Acta Pharm 2014; 64(2):223-32. doi: http://dx.doi. org/10.2478/acph-2014-0020

18. Urquhart BL, Kim RB. Blood-brain barrier transporters and response to CNS-active drugs. Eur J Clin Pharmacol 2009; 65(11):1063-70. doi: http:// dx.doi.org/10.1007/s00228-009-0714-8

19. Patel M, Souto EB, Singh KK. Advances in brain drug targeting and delivery: limitations and challenges of solid lipid nanoparticles. Expert Opin Drug Deliv 2013; 10(7):889-905. doi: http:// dx.doi.org/10.1517/17425247.2013.784742

20. Zhang R, Jie J, Zhou Y, Cao Z, Li W. Longterm effects of Panax ginseng on disposition of fexofenadine in rats in vivo. Am J Chin Med 2009; 37:657-667. doi: http://dx.doi.org/ 10.1142/S0192415X09007144

21. Zhang J, Zhou F, Wu X, Gu Y, Ai H, Zheng Y, et al. 20(S)-ginsenoside Rh2 noncompetitively inhibits P-glycoprotein in vitro and in vivo: a case for herb-drug interactions. Drug Metab Dispos 2010; 38:2179-2187. doi: http://dx.doi. org/10.1124/dmd.110.034793 
22. Garrovo Ch, Rosati A, Bartoli F, Decorti G. St John's wort modulation and developmental expression of multidrug transporters in the rat. Phytother Res 2006; 20:468-473. doi: http:// dx.doi.org/10.1016/j.taap.2004.04.020

23. Scambia G, Ranelletti FO, Panici PB, De Vincenzo R, Bonanno G, Ferrandina G, et al. Quercetin potentiates the effect of adriamycin in a multidrug-resistant MCF-7 human breast-cancer cell line: P-glycoprotein as a possible target. Cancer Chemoth Pharm 1994; 34:459-464. doi: http:// dx.doi.org/ 10.1371/journal.pone.0051764

24. Yuan J, Liu W, Zhu H, Zhang X, Feng Y, Chen Y, et al. Curcumin attenuates blood-brain barrier dis- ruption after subarachnoid hemorrhage in mice. J Surg Res 2017; 207:85-91. doi: http://dx.doi.org/ 10.1016/j.jss.2016.08.090

25. Wang YF, Gu YT, Qin GH, Zhong L, Meng YN. Curcumin ameliorates the permeability of the blood-brain barrier during hypoxia by upregulating heme oxygenase-1 expression in brain microvascular endothelial cells. J Mol Neurosci 2013; 51(2):344-351. doi: http://dx.doi.org/10.1007/ s12031-013-9989-4

26. Klinger NV, Mittal S. Therapeutic potential of curcumin for the treatment of brain tumors. Oxid Med Cell Longev 2016; 2016:9324085. doi: http:// dx.doi.org/ 10.1155/2016/9324085 